\title{
Enquête sérologique sur les Rickettsioses chez le dromadaire au Tchad *
}

\author{
Y. MAURICE, J.-F. BARES, et Mme BAILLE
}

\begin{abstract}
RÉSUMÉ
Les auteurs ont examiné en utilisant la réaction de microagglutination sur lame suivant la technique de GIROUD, 500 échantillons de sérums de dromadaires de différentes régıons du Tchad dans le but de détecter la présence éventuelle d'anticorps contre les antigènes rickettsiens majeurs et l'antigène de la souche $Q 18$ de néorickeltsie. Les résultals rapportés sur les tableaux en annexe mettent en évidence la rareté des anticorps antiépidémique et antiboutonneux. Par contre un certain nombre de sérums réagissent positivement vis-à-vis de l'antigène murin tandis que d'autres répondent positivement à Ricketisio burneti et à la souche $Q 18$ de néorickettsie.
\end{abstract}

Le problème de l'épidémiologie des rickettsioses a été étudié jusqu'ici principalement chez I'homme, les animaux sauvages et les animaux domestiques. Parmi ceux-ci les chevaux, les caprins, les ovins et les bovins ont surtout retenu l'attention des épidémiologistes. Le comportement sérologique de ces espèces animales vis-àvis de Rickettsia prowazeki, Rickettsia mooseri, Rickettsia conori, Rickettsia burneti et Néorickettsie a fait l'objet depuis plusieurs années d'un nombre considérable d'enquêtes et de travaux. Ainsı il nous a été donné l'occasion ces dernières années d'étudier l'incidence des rickettsioses et néorickettsioses chez les bovins ef les petits ruminants d'Afrique centrale (12) (13) (14) (15) et en particulier chez ceux du Tchad (6). Cependant à notre connaissance aucun travail d'ensemble n'a été entrepris jusqu'ici chez le dromadaire. Seuls les anticorps antiburneti ou antiprowazeki ont été recherchés séparément chez le chameau ou le dromadaire (3) (4) (5) (10) (11) (16) (17). Il étalt donc intéressant, quand on pense à l'association étroite existant entre

* Communication présentée au 18 e congrès mondial vétérinaire, Paris, 17-22 juillet 1967. l'homme et le dromadaire dans certaines régions d'Afrique et en particulier chez certaines populations du Tchad, d'effectuer chez cette espèce animale une enquête poriant sur un nombre relativement élevé d'échantillons de sérums provenant de régions géographiques différentes, en analysant ceux-ci vis-à-vis des 5 antigènes majeurs : Rickettsıa prowazeki, Rickettsia mooseri, Rickettsia conori, Rickettsia burneti, et la souche Q 18 de Néorickettsie.

\section{MATÉRIEL ET MÉTHODE}

I) Sérums : 500 sérums de dromadaires adultes en bonne santé ont été examinés. La provenance de ces sérums est la suivante :

\begin{tabular}{|c|c|}
\hline Mao & \\
\hline Nokou & \\
\hline Moussoro & \\
\hline Zigueï .. & \\
\hline Massakory & $\ldots \ldots \ldots \ldots$ \\
\hline Fort-Lamy & $\ldots \ldots \ldots \ldots$ \\
\hline Ati,$\ldots .$. & $\ldots \ldots \ldots \ldots \ldots$ \\
\hline Abéché & \\
\hline
\end{tabular}

II) Antigènes ef techniques utilisées : Cette 


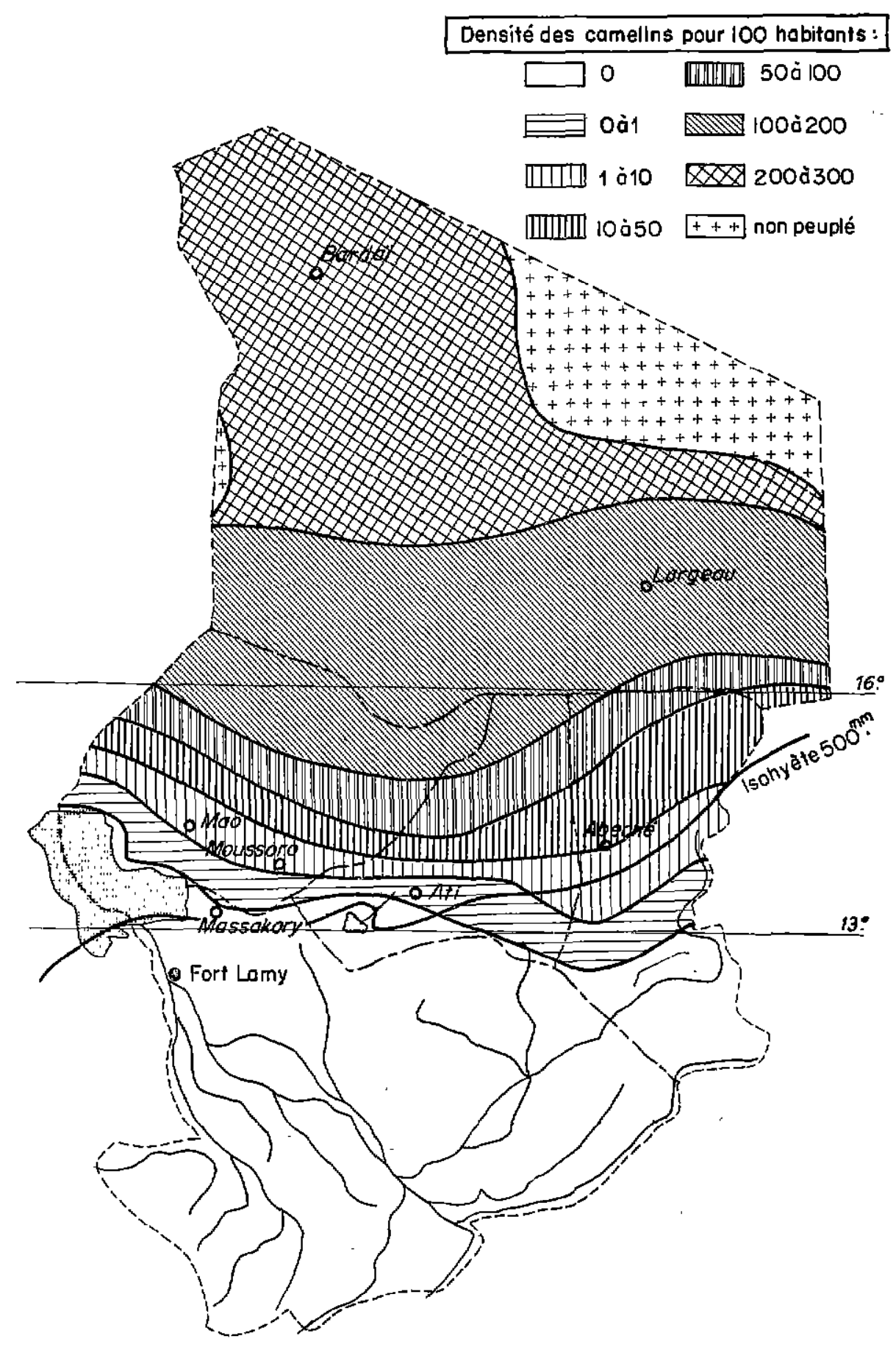


enquête a été effectuée en utilisant la réaction de micraagglutination sur lame de GIROUD. En effet, depuis que BABUDIERI en 1953 (1) a confirmé la valeur de cetfe réaction et que en 1959 WELSH et ses collaborateurs (21) ont reconnu en examinant 1.017 sérums que le test d'agglutination était supérieur au trois autres tests à savoir la fixation du complément, l'agglutination macroscopique et l'agglutination en tube capillaire, cette technique s'est révélée être la méthode de choix par sa sûreté et sa précision qui sont maintenant bien établies.

Les suspensions des divers types de rickettsies préparées par l'Institut Pasteur (Service de M. Paul GIROUD) ont été utilisées dans cette réaction. Les sérums ont été éprouvés vis-à-vis des souches épidémiques (taux 1/320), murine (taux 1/160), boutonneuse (taux 1/160), fièvre $Q$ (taux 1/20), ef $Q 18$ (taux 1/20). Seules les agglutinations nettement positives ont été retenueș. Certaines d'entre elles ont été vérifiées en dilutions supérieures mais on n'a pas cherché à établir un taux limite de positivité.

\section{RÉSULTATS ET COMMENTAIRES}

\section{A) Résultats :}

568 réactions ont été effectuées au titre de cette enquête sur les sérums de dromadaire.

10 Réactions qualitatives :

500 réactions ont été effectuées. Les résultats sont les suivants :

- sérums positifs vis-à-vis de l'un ou de plusieurs antigènes : 102 soit 20,4 p. 100 :

- sérums douteux vis-à-vis de l'un ou de plusieurs des cing antigènes avec absence de réaction positive vis-à-vis de l'un des cinq antigènes : 4 soit 0,8 p. 100 .

- sérums négatifs vis-à-vis des cinq antigènes: 394 soit 78,8 p. 100 ;

La répartition des sérums positifs vis-à-vis de chacun des cinq antigènes est indiquée dans les tableaux $I$ ef II. Le tableau I correspond aux résultats globaux, le tableau II tient compte de l'origine géographique des sérums.

TABLEAU N ${ }^{\circ} \mathrm{I}$

Résultats globaux

\begin{tabular}{|c|c|c|}
\hline Antigène & Sérums positifs & Sếrums douteux \\
\hline Epidémique & 9 soit $1,8 \mathrm{p} .100$ & 0 \\
\hline Murin & 58 soit $11,6 \mathrm{p} .100$ & 3 soit $0,6 \mathrm{p} .100$ \\
\hline Boutonneux & 5 soit 1 p.100 & 2 soit $0,4 \mathrm{p} .100$ \\
\hline Fièvre Q & 24 soit $4,8 \mathrm{p} .100$ & 19 soit $3,8 \mathrm{p} .100$ \\
\hline Néo Q 18 & 27 soit $5,4 \mathrm{p} .100$ & 0 \\
\hline
\end{tabular}

TABLEAU N*II

Orıgine géographique des sêrums

\begin{tabular}{|c|c|c|c|c|c|c|}
\hline Localité & Sérums & Epldémique & Plurin & Boutonneux & Fièvre Q & Nêo Q 18 \\
\hline Mao & 131 & 1 & 7 & $2-$ & 2 & 18 \\
\hline Moussora & 129 & 4 & 31 & 3 & 19 & 6 \\
\hline Fort-Lamy & 84 & 0 & 5 & 0 & 0 & 1 \\
\hline Abuêchể & 93 & 3 & 10 & 0 & 0 & 0 \\
\hline Massakory & 1 & 0 & 0 & 0 & 0 & o \\
\hline Zigue $\bar{x}$ & 34 & 1 & 5 & 0 & 1 & 2 \\
\hline Nokou & $\cdots 23$ & 0 & 0 & 0 & 2 & 0 \\
\hline Ati & 5 & 0 & 0 & 0 & 0 & 0 \\
\hline Totaux & 500 & $9-$ & 58 & 5 & 24 & 27 \\
\hline
\end{tabular}


Quelques sérums ont été positifs simultanément vis-à-vis de deux ou plusieurs antigènes. Les seules combinaisons qui se sont trouvées réalisées sont celles rapportées sur le tableau III.

\section{TABLEAU $N^{\circ}$ III}

Sérums positifs simultanément vis à vis de deux ou plusieurs antigènes

\begin{tabular}{|c|c|c|}
\hline Antigène & Positif & Douteux \\
\hline Epidëmique (E.) & 1 & \\
\hline Murin (M.) & 43 & $I$ \\
\hline Fiêvre Q (Q) & 20 & 17 \\
\hline Routonneux (B.) & 1 & \\
\hline $\begin{array}{l}\text { Néorickettsien } \\
\text { Q } 1 \text { (N) }\end{array}$ & 20 & \\
\hline E.M. & 6 & \\
\hline M.B. & 1 & (1) $M+B \pm$ \\
\hline M.Q. & 2 & \\
\hline M.N. & 2 & (1) $M+N+M \pm$ \\
\hline$Q \cdot N$. & 1 & \\
\hline E.Q.N. & 1 & \\
\hline M.B.Q. & & (1) $M+B+Q \pm$ \\
\hline E.M.B.N. & & (1) $E+M+B+N+$ \\
\hline M.B.Q.N. & & (1) $M+B+Q \pm N+$ \\
\hline
\end{tabular}

20 Réactions quantitatives:

68 réactions quantitatives ont été effectuées.

- Antigène épidémique : 6 réactions : 5 sérums positifs au 1/640, 1 au 1/320.

- Antigène Murin : 35 réactions : 4 sérums positifs au 1/1.280, 1 au 1/640, 12 au $1 / 320$, 18 au $1 / 160$.

- Antigène Fièvre $Q: 16$ réactions : 2 sérums positifs au 1/160, 5 au 1/80, 3 au 1/40, 6 au 1/20.

- Antigène Néo $Q 18: 11$ réactions : 2 sérums positifs au 1/320, 5 au 1/80, 1 au 1/40, 3 au 1/20.

\section{B) Commentaires :}

1020,4 p. 100 des animaux ont une sérologie rickettsienne ou néorickettsienne positive. Le pourcentage de sérologie positive est dans l'ensemble moins élevé que celui des autres animaux domestiques (bovins, ovins, caprins, chevaux) vivant dans les mêmes régions (13), il est cependant appréciable. Le tableau IV est donné à titre de comparaison.

20 1,8 p. 100 seulement des sérums étudiés sont positifs vis-d-vis de l'antigène du typhus épidémique. Les résultats obtenus chez les bovins, les ovins, les caprins et les équins dans la région de Mao, Moussoro, Abéché sont du même ordre de grandeur (tableau $V$ ). Parmi ces 9 sérums positifs l'un d'eux l'est vis-à-vis du seul antigène épidémique, 7 des autres le sont également visà-vis de l'antigène murin. Les analyses quantitatives effectuées sur 6 de ces sérums ont donné

TABLEAU $\mathrm{N}^{\circ} \mathrm{IV}$

Pourcentages comparés selon les espèces des animaux domestiques à sêrologie rickettsienne et néorickettsienne posttive

\begin{tabular}{|c|c|c|c|}
\hline Espèces & Mao & Moussaro & Abēchë \\
\hline Chameaux & $\begin{array}{c}24 \text { sur } 131 \\
\text { soit } 18,32 \text { p.100 }\end{array}$ & $\begin{array}{c}52 \text { sure } 129 \\
\text { soit } 40,31 \text { p.100 }\end{array}$ & $\begin{array}{l}11 \text { sut } 93 \\
\text { soit } 11,83 \mathrm{p} .100\end{array}$ \\
\hline Chềvres & $\begin{array}{c}13 \text { sur } 36 \\
\text { soit } 36,11 \text { p.100 }\end{array}$ & $\begin{array}{l}31 \text { sur } 86 \\
\text { soit } 36,04 \text { p.100 }\end{array}$ & $\begin{array}{l}14 \text { sur } 27 \\
\text { soit } 51,85 \text { p.100 }\end{array}$ \\
\hline Moutans & $\begin{array}{c}9 \text { sur } 26 \\
\text { soit } 34,62 \text { p.100 }\end{array}$ & & $\begin{array}{c}15 \text { sur } 29 \\
\text { soit } 51,72 \text { p.100 }\end{array}$ \\
\hline Chevaux & $\begin{array}{c}10 \text { sur } 30 \\
\text { soit } 33,33 \text { p. } 100\end{array}$ & $\begin{array}{cl}3 \text { sur } B \\
\text { soit } 37,5 & \text { p.100 }\end{array}$ & $\begin{array}{l}12 \text { sur } 14 \\
\text { soit } 85,71 \text { p. } 100\end{array}$ \\
\hline Bovins & $\begin{array}{c}21 \text { sur } 29 \\
\text { soit } 72,41 \text { p. } 100\end{array}$ & $\begin{array}{cc}78 \text { sur } & 100 \\
\text { soit } 78 & \text { p. } 100\end{array}$ & $\begin{array}{l}21 \text { sur } 72 \\
\text { soit } 29,17 \text { p.100 }\end{array}$ \\
\hline
\end{tabular}




\begin{tabular}{|c|c|c|c|c|c|c|c|c|c|c|c|c|c|c|c|c|}
\hline $\begin{array}{l}8 \varepsilon^{\mathrm{c} T} \mathrm{~T} \\
\angle L^{\mathrm{C}} \angle Z\end{array}$ & $\begin{array}{l}0 \\
\text { I } \\
0 z\end{array}$ & $\begin{array}{l}Z L \\
Z L \\
Z L\end{array}$ & $\begin{array}{l}8 Z^{6} 7 \tau \\
\tau L^{c} 58\end{array}$ & $\begin{array}{l}0 \\
Z \\
\tau I\end{array}$ & $\begin{array}{l}\eta \mathrm{II} \\
\forall \mathrm{I} \\
\forall \mathrm{I}\end{array}$ & $Z L^{6} I S$ & $\begin{array}{l}0 \\
0 \\
\text { SI }\end{array}$ & $\begin{array}{l}62 \\
62 \\
62\end{array}$ & $\begin{array}{l}7 \tau^{6} L \\
\tau L^{\circ} 0 \pi \\
L^{\circ} 09\end{array}$ & $\begin{array}{l}Z \\
\varepsilon \\
L I\end{array}$ & $\begin{array}{l}87 \\
87 \\
87\end{array}$ & SL'OT & $\begin{array}{l}0 \\
0 \\
\text { OI }\end{array}$ & $\begin{array}{l}\varepsilon 6 \\
\varepsilon 6 \\
\varepsilon 6\end{array}$ & 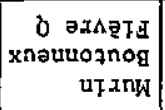 & จุจจุจ \\
\hline $\begin{array}{l}z \\
0 z \\
5 L\end{array}$ & $\begin{array}{l}z \\
0 z \\
s L\end{array}$ & $\begin{array}{l}\text { OOt } \\
00 \mathrm{~T} \\
00 \mathrm{I}\end{array}$ & $\begin{array}{l}s^{\prime} Z \mathrm{Z} \\
\mathrm{s}^{\prime} \angle \varepsilon\end{array}$ & $\begin{array}{l}o \\
\tau \\
\varepsilon\end{array}$ & $\begin{array}{l}8 \\
8 \\
8\end{array}$ & & & & $\begin{array}{l}\angle 6^{\circ} 9 \\
9 T^{\prime} 7 \\
88^{\circ} \mapsto \varepsilon\end{array}$ & $\begin{array}{l}9 \\
\text { I } \\
0 \varepsilon\end{array}$ & $\begin{array}{l}98 \\
98 \\
98\end{array}$ & $\begin{array}{l}O L{ }^{\prime} \eta \tau \\
z \varepsilon^{\prime} z \\
\varepsilon O^{\prime} \eta z\end{array}$ & $\begin{array}{l}6 I \\
E \\
I \varepsilon\end{array}$ & $\begin{array}{l}6 Z I \\
6 Z I \\
6 Z I\end{array}$ & 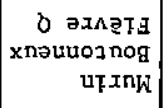 & oxosenoy \\
\hline $\begin{array}{l}\varepsilon 0^{\circ} \mathrm{IE} \\
\angle 0^{\circ} \mathrm{Zg}\end{array}$ & $\begin{array}{l}0 \\
6 \\
8 I\end{array}$ & $\begin{array}{l}62 \\
62 \\
62\end{array}$ & $\varepsilon \varepsilon^{*} \varepsilon \varepsilon$ & $\begin{array}{l}0 \\
0 \\
\text { ot }\end{array}$ & $\begin{array}{l}\text { OE } \\
O E \\
O E\end{array}$ & $\begin{array}{l}s 8^{\prime} \varepsilon \\
L L^{\prime} O E\end{array}$ & $\begin{array}{l}0 \\
I \\
8\end{array}$ & $\begin{array}{l}92 \\
92 \\
92\end{array}$ & $\tau \tau^{\prime} 9 \varepsilon$ & $\begin{array}{l}0 \\
0 \\
\varepsilon I\end{array}$ & $\begin{array}{l}9 \varepsilon \\
9 \varepsilon \\
9 \sqsubset\end{array}$ & $\begin{array}{l}\varepsilon S^{*} I \\
E S^{*} L \\
z \hbar^{*} S\end{array}$ & $\begin{array}{l}z \\
z \\
l\end{array}$ & $\begin{array}{l}I \in I \\
I \varepsilon \tau \\
I \in T\end{array}$ & 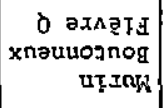 & OEW \\
\hline $00 \tau \cdot d$ & 8ร7วT80ة & $\begin{array}{l}\text { SmnIอs } \\
\text { əxquoN }\end{array}$ & $00 T \cdot d$ & geftzisod & $\begin{array}{l}\text { smnבes } \\
\text { axquoN }\end{array}$ & $00 \pi \cdot d$ & sft7t sod & 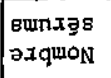 & $00 \tau \cdot d$ & S孔Fनf50d & $\begin{array}{l}\text { sunzogs } \\
\text { əaquoN }\end{array}$ & $00 T^{\cdot} d$ & stfafsod & $\begin{array}{l}\text { sunxass } \\
\text { aIquoN }\end{array}$ & \multirow[t]{2}{*}{ auอ8 } & \multirow[t]{2}{*}{ อวนBนอ $\Lambda O I_{d}$} \\
\hline \multicolumn{3}{|c|}{ Вันุดg } & \multicolumn{3}{|c|}{$x n-3 \wedge \approx 40$} & \multicolumn{3}{|c|}{ вwoznow } & \multicolumn{3}{|c|}{ 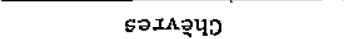 } & \multicolumn{3}{|c|}{ хпвәшвЧว } & & \\
\hline
\end{tabular}

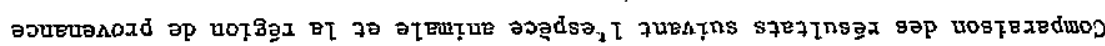

$\Lambda_{0} N$ กษดTgVน 
respectivement pour les antigènẹs épidémiqué et murin les taux d'agglutination suivants :

(No $1: 1 / 640,0 ;$ No $2: 1 / 640,1 / 160 ;$ No 3 : $1 / 640,1 / 320 ;$ No $4 ; 1 / 640,1 / 1280 ;$ No $5: 1 / 640$, $\left.1 / 1.280 ; N^{\circ} 6: 1 / 320,1 / 160\right)$.

La question qui se pose est la suivante : le dromadaire qui réaglt vis-à-vis de Rickettsia prowazeki est-il capable de conserver la souche épidémique (17) (18) (19) ? Est-il seulement le témoin de l'infection des tiques? Ou bien, assistons-nous chez lui à la transformation de certains types d'antigènes puisqu'en effet on sait qu'il y a communauté antigénique entre certaines souches comme Rickettsia prowazeki, Rickettsia mooseri, Rickettsia conori (7) ? Autant de questions auxquelles il est difficile de répondre. Rappelons à ce propos que récemment IMAN et ALFI (10) en Egypte ont trouvé 14,1 p. 100 de sérums de chameaux positifs vis-à-vis de Rickettsia prowazeki mais n'ont pu isoler de souches de rickettsies à partir du sang des animaux.

$3^{\circ}$ Les dromadaires des régions de Mao, Moussoro, Fort-Lamy, Abéché, Zıgueï répondent positivement à l'antigène murin et parfois à des taux élevés. Ceci n'est pas pour nous étonner. Il a été constaté en effet après 3 années d'enquête sur les sérums d'animaux domestiques et sauvages en Afrique centrale que la positivité des sérums vis-à-vis de Rickettsia mooseri constituait la dominante des réactions positives en matière de sérologie des rickettsioses majeures (13). Le chiffre de 11,6 p. 100 est cependant dans l'ensemble inférieur à celui obtenu chez les autres espèces domestiques dans les mêmes régions (tableau V), 43 des 58 sérums positifs le sont exclusivement vis-à-vıs de l'antigène murin. II semblerait donc que l'agent du typhus murin puisse infecter de façon inapparente le dromadaire, l'infection étant révélée par les anticorps presque exclusivement antimurin de leurs sérums.

40 5 animaux seulement, soit 1 p. 100 possèdent des anticorps antiboutonneux. Parmi ceux-ci, un seul est positif vis-à-vis de Rickeftsia conori exclusivement. Le tableau $V$ montre que suivant les" espèces et les localités considérěes les résultats peuvent être variables.

5o 24 des sérums, soit 4,8 p. 100 sont positifs vis-d-vis de l'antigène de la Fièrre $Q$. Ce pourcentage est du même ordre de grandeur que celui obtenu chez les bovins et les autres animaux domestiques dans les mêmes régions (15) (tableau $V$ ). Mais on remarque que 16 de ces 24 sérums correspondent à des animaux vivant dans le même troupeau de 56 têtes, c'est-à-dire que 28,57 p. 100 de l'effectif considéré montre la trace d'uhe infection do Rickettsia burneti, ancienne ou actuelle et silencieuse, ou les deux à la fois, les animaux de ce troupeau 'n'extériorisant en effet aucun symptôme. Cette observation complète les résultats obtenus lors de nos précédentes enquâtes sur la Fièvre $Q(15)$ au cours de laquelle le rôle du dromadaire dans l'épidémiologie de cette zoonose n'avait pu à l'époque être mis en évidence. II est utile de rappeler à ce sujet que c'est en 1948 que BLANC et collaborateurs (2) ont démontré au Maroc la sensibilité du dromadaire à la Fièvre $\mathrm{Q}$ en infectant expérımentalement l'un d'eux avec Rickettsia burneti. En 1954, GIROUD et collaborateurs (8) ont constaté que deux sérums de dromadaires du Tchad sur 9 analysés montraient des traces d'anticorps VIs-à-vìs de l'antigène de la fièvre $Q$. La même année en Iran, RAYFI et MAGHAMI (16) ont décelé sérologiquement cette infection à partir de 2 sérums de dromadaires sur 12 étudiés. ELYAN et DAWOOD (1954) (5) en Egypte, IMAMOV (1962) (11) en Asie centrale ont pu faire les mêmes constatations ainsi que BROWN (1956) (3) au Kenya qui a noté que 4 sérums de chameaux sur 20 étudiés présentaient des anticorps déviant le complément vis-à-vis de la souche américaine Nine Mile. Le dromadaire intervient donc également au Tchad dans l'épidémiologie de la Fièvre $Q$. Il était intéressant de le signaler et de montrer surtout que certains troupeaux pouvaient être lourdement infectés.

60 27 sérums soit 5,4 p. 100 se monirent positifs vis-d̀-vis de la souche $Q 18$ de néorickettsie. Parmi ceux-ci on relève (tableau II) 18 sérums de Mao et 6 de Moussoro. On serait tenté de tirer une conclusion faisant intervenir le rôle du facteur géográphique. Ainsi les néorickettsies du groupe Q 18 seraient présentes dans la région de Mao et de Moussoro, pratiquement absentes à Abéché ef Fort-Lamy. Une telle conclusion serait sans vैaleur, tel sérum prélevé à Fort-Lamy pouvant très bien provenir d'un animal venant de Mao Moussoro oy Abéché et inversement. En effet les mouvements des dromadaires dans le nord et l'est du Tchad sont variés. Ainsi les Goranes 
anakasas de Faya-Largeau descendent avec leurs dromadaires à Moussoro pour livrer des dattes et remonter du mil ; cerfains chameliers de Moussoro vont jusqu'à Zouar situé au nord de Faya et se déplacent bien au-delà des frontières de l'état tchadien en passant au Niger par le nord du lac Tchad. Sans préjuger de l'incidence relative des néorickettsioses dans ces régions, il est permis de penser que les néorickettsioses sont une composante de la pathologre du dromadaire et interviennent vraisemblablement dans le complexe «Affections pulmonaires du dromadare ». II est à remarquer que seule la souche Q 18 de Néorıckettsies a été utilisée : certains sérums qui ont réagi négativement vis-à-vis de cette souche auraient peut-être été positifs vis-d̀-vis d'une souche différente de Q 18.

Sur les 27 sérums positifs, 24 le sont vis-à-vis de la souche $Q 18$ seule ou simultanément visd̀-vis de la souche $Q 18$ et d'un autre antigène, différent de celui de la Fièvre $Q$. C'est donc un total de 48 animaux soit 9,6 p. 100 qui présentent une infection $Q$ ou $Q 18$, résultat qui a son importance du point de vue de la pathologie comparée. (II n'a pas été tenu compte des sérums présentant des traces d'anticorps).

\section{CONCLUSION}

10 20,4 p. 100 des dromadaires du Tchad ont une sérologie rickettsienne ou néorickettsienne positive. L'isolement des souches reste à faire.
Le pouvoir pathogène de celles-ci est inconnu. II est logique de penser que le cheptel camelin du Tchad est atteint des mêmes affections rickettsiennes et néorickettsiennes que les bovins se manifestant notamment par des avortements, des pneumonies, des maladies inapparentes, etc... II est vraisemblable que I'homme vivant en contact étroit avec le dromadaire en subisse les conséquences sous forme de maladies très diverses ; vasculaires, teratogènes, oculaires, manifestations méningées et exanthématiques, manifestations fébriles pseudogrippales, affections pulmonaires diverses, avortements, etc...

$2^{\circ}$ Les résultats obtenus confirment les travaux antérieurs qui ont montré que le chameau possède des anticorps antiépidémiques et intervient vraisemblablement dans l'épidémiologie de la Fièvre $Q$. Ils montrent également que les effectifs de dromadaires au Tchad peuvent dans certains cas être lourdement infectés par Rickettsio burneti.

$3^{\circ}$ Le dromadaire est le témoin d'une infection à Rickettsıa mooseri et à Néorickeftsıa souche Q 18. II. intervient vraisemblablement dans l'épidémiologie du typhus murin et des néorickettsioses. II serait intéressant de préciser le rôle des néorickettsies dans le complexe « affection pulmonaires du dromadaire $»$.

\footnotetext{
Institut d'Elevage et de Médecine vétérinaire des Poys tropicaus Laboratare de Forcha - Fort-Lamy et Service de l'Elevage du Tchad.
}

\section{SUMMARY \\ Serological survey on the Rickettsioses in Dromedary in Chad}

500 samples of serum of dromedary from various areas in Chad, have been investıgated by the slide microagglutination test, according Giroud's method, in order to evidence antıbodies against usual Rickettsiae and the strain $Q 18$ of Neoricketisıa.

The results recorded on the tables show that the antibodies against epidemic and pustulous Rickettsiosis are rarely evidenced. On an other hand, some sera show a positive reaction against murıne antigen and some others are positive to Rickettsio burneti and to the strain Q18 of Neorickettsio.

\section{RESUMEN}

Encuesta serológica sobre las rickettsiosis en el dromedario del Chad

Utilizando la reacción de micraaglutinación sobre lamina según la técnica de GIROUD, los autores examinaron 500 muestros de sueros de dromedarios de varias regiones de Chad con el objeto de descubrir la presencia eventual 
de anticuerpos contra los principales antigenos rickettsianos y el antigeno de la cepa $Q 18$ de neorickettsia.

Los resultados notados en los cuadros anejos demuestran la rareza de los anticuerpos antiepidémicos y antibotonosos. En cambio un cierto número de sueros reaccionan positivamente para con el antigeno murino mientras otros reaccionan positivamente para con Rickettsio burneti y la cepa $Q 18$ de neorickettsia.

\section{BIBLIOGRAPHIE}

1. BABUDIERI (B.).-Monographie OMS, 1953, no $19: 193$.

2. BLANC (G.), BRUNEAU (J.), MARTIN (J.A.), MAURICE (A.). - Quelques données nouvelles sur le virus de la $Q$. Fever marocaine. C. R. Acad. Sci., 1948, $226: 607$.

3. BROWN (R. D.), - La mise en évidence par tests sérologiques de la Fièvre $Q$ chez les animaux domestiques du Kenya. Bull. Epiz. Dis. Afric., 1956, 4 : 115-119.

4. EL NASRI (M.). - A serological survey for the detection of $Q$ Fever antibodies in the sera of animals in the Sudan. Bull. Epiz. Dis. Afric., 1962, 10, 55-57.

5. ELYAN (A.) et DAWOOD (M. M.). - J. Egypt. publ. Hith. Ass., 1954, $29: 185$.

6. GIDEL (R.). — Contribution à l'étude des Rickettsioses au Tchad. Enquête épidémiologique. Rev. Elev. Méd. Vét. Pays trop., $1965,18,2: 127-136$.

7. GIROUD (P.), LE GAC (P.), BRIZARD (H.), LAURENT (C.). - Comportement des sérums de divers animaux domestiques de l'Oubangui-chari vis-à-vis de l'antigène épidémique. Bull. Soc. Path. Exot, 1952, 45 : 313.

8. GIROUD (P.), ROGER (F.), DUMAS (N.), VOUILLOUX (P.), SACQUET (E.). — Comportement des animaux domestiques de la région du Tchad vis-à-vis de l'antigène $T_{3}$. Bull. Soc. Path. Exot., 1954, 47 : 644-645.

9. GRUVEL (J.) et GRABER (M.). - Quelques résultats d'enquêtes récentes sur la globidiose du dromadaire au Tchad. Rev. Elev. Méd. Vét. Pays trop., 1965, 18, 4 : p. 423.

10. IMAM (Z. E.), IMAM et ALFY (L.). - Evidence of typhus infection in domestic animals in Egypt. Bull. O. M. S., 1966, 35 : 123-126.

11. IMAMOV (E. D.). - La Fièvre $Q$ chez les animaux domestiques de Kirghizie, Frunze. (Cité par P. F. ZORODOVSKIJ dans «les rickettsioses en U. R. S. S. ») Bull. O.M. S., $1964,31: 33-43$.

12. MAURICE (Y.). - Contribution à l'étude des Rickettsioses en République Centrafricaine. Enquête épidémiologique (à paraître).

13. MAURICE (Y.). - Consiatations sérologiques vis-à-vis des antigènes rickettsiens chez les animaux d'Afrique centrale (en préparation).

14. MAURICE (Y.), CHAMOISEAU (G.), VIGIER (M.), PROVOST (A.). - Contribution à l'étude des avortements au Nord-Cameroun (Zoonoses rickettsiennes, neorickettsiennes et brucelliennes des bovins) (en préparation).

15. MAURICE (Y.) et GIDEL (R.). - Incidence de la Fièvre $Q$ en Afrique Centrale (à parâ̂tre).

16. RAFYI (A.) et MAGHAMI (C.), - Sur la présence de la Fièvre $Q$ en Iran. Bull. Soc. Path. Exot., 1954, no $6: 766$.

17. REISS-GUTFREUNDT (R.). - - Isolement de souches de Rickettsia prowazeki à partir du sang des animaux domestiques d'Ethiopie et de leurs tiques. Bull. Soc. Poth. Exot., 1955. 48, p. 602.

18. REISS-GUTFREUNDT (R.). - Un nouveau réservoir de virus pour Rickettsia prowazeki : les animaux domestiques ef leurs tiques. Bull. Soc. Path. exot., 1956, 49, 946.

19. REISS-GUTFREUNDT (R.). - Nouveaux isolements de Rickettsia prowazeki à partir d'animaux domestiques et de tiques. Bull. Soc. Path. Exot., 1961, 54, 284.

20. RIZK (E.). - Q Fever complement fixing antibodies in animal and human sera in the Middle East. Lebanon. Med. J., 1954, 7, 226-235.

21. WELSH $(H . H),. J E N S E N(F . W$.$) et LEN-$ NETTE (E. H.), - Americ. J. of Hyg., 1959, 70, p. 1. 